Z Rheumatol 2006 ·65:92-92 DOI 10.1007/s00393-006-0048-1

Online publiziert: 28. Februar 2006

(c) Springer Medizin Verlag 2006

J. Smolen

Universitätsklinik für Innere Medizin III, Abt. für Rheumatologie, Wien

\title{
Endpunkt- und Verlaufsmessungen bei entzündlichen rheumatischen Erkrankungen in Studien und Praxis
}

Sehr geehrte Leserin, sehr geehrter Leser,

Das Jahrzehnt zwischen 1995 und 2005 hat der Rheumatologie großartige Umbrüche beschert. Die wohl wichtigste Entwicklung bestand in der Etablierung neuer, höchst effizienter Therapien für die rheumatoide Arthritis, wobei zum einen die hochdosierte Methotrexat-Behandlung, zum anderen die Einführung der Biologika, insbesondere der TNF-Blocker, von wesentlicher Bedeutung waren. Die TNF-Blocker haben aber auch die Behandlung der Psoriasisarthritis und des Morbus Bechterew revolutioniert, zumal gerade für die ankylosierende Spondylitis bis dahin keine Basistherapie verfügbar war.

\section{$>$ Aktivität, Funktion und therapeutisches Ansprechen werden in der Rheumatologie oft nicht regelmäßig erfasst}

Parallel $z u$ diesen Entwicklungen der Grundlagen- und industriellen Forschung sind aber auch in der klinischen Forschung neue Einsichten entstanden, ohne die viele der mit den neuen Therapien erzielten Erfolge nicht hinreichend messbar und damit wohl nur unzureichend erfassbar gewesen wären. Während aber die neuen Therapien rasch Eingang in die Praxis gefunden haben, scheint die Bedeutung der regelmäßigen Ermittlung von Krankheitsaktivität und -progredienz bei Patienten mit entzündlichen rheumatischen Erkrankungen noch nicht vollständig in unser Bewusstsein gedrungen zu sein.

Und gerade auf diesem Aspekt liegt der Themenschwerpunkt dieses Heftes. Wäh- rend in anderen Fachbereichen wie etwa der Kardiologie oder Endokrinologie/ Diabetologie die Erfassung von Aktivität, Funktion und therapeutischem Ansprechen verschiedener Organerkrankungen regelmäßig vorgenommen wird, ist dies in der Rheumatologie vielfach nicht der Fall - in unserem Bereich sind es eben meist die Gelenke oder die Wirbelsäule, die als „Organe" betroffen sind.

- Welche Messmethoden der Krankheitsaktivität bei rheumatoider Arthritis, Psoriasisarthritis, ankylosierender Spondylitis und systemischem Lupus erythematosus stehen zur Verfügung?

- Weshalb sind diese "Messungen" so wichtig?

- Welche Vorteile bieten sie?

Diesen Fragen widmen sich eine Reihe von Experten, die die Entwicklung des Fachgebietes in diesen letzten zehn Jahren wesentlich beeinflusst haben, im Rahmen ihrer Beiträge, in denen Hintergrundinformation, Formeln und praktische Einsatzmöglichkeiten dargelegt werden.

Die Umsetzung dieser Erkenntnisse im Alltag kann zu einer wesentlichen Verbesserung der Patientenbetreuung und der Therapieerfolge führen.

J. Smolen 